\title{
Household latrine utilization and its association with educational status of household heads in Ethiopia: a systematic review and meta-analysis
}

Cheru Tesema Leshargie ${ }^{1 *}$, Animut Alebel ${ }^{2}$, Ayenew Negesse $^{3}$, Getachew Mengistu $^{4}$, Amsalu Taye Wondemagegn ${ }^{5}$, Henok Mulugeta ${ }^{2}$, Bekele Tesfaye ${ }^{2}$, Nakachew Mekonnen Alamirew ${ }^{1}$, Fasil Wagnew², Yihalem Abebe Belay ${ }^{1}$, Aster Ferede ${ }^{1}$, Mezinew Sintayehu², Getnet Dessie ${ }^{2}$, Dube Jara Boneya ${ }^{1}$, Molla Yigzaw Birhanu and Getiye Dejenu Kibret ${ }^{1}$

\begin{abstract}
Background: Ethiopia has been experiencing a high prevalence of communicable diseases, which resulted in high morbidity, mortality, and hospital admission rates. One of the highest contributing factors for this is lower level of latrine utilization. There had been significantly varying finding reports with regard to the level of latrine utilization and its association with education level from different pocket studies in the country. Therefore, this systematic review and meta-analysis was aimed to estimate the pooled prevalence of household latrine utilization and its association with education status of household heads, in Ethiopia using available studies.
\end{abstract}

Methods: This systematic review and meta-analysis was conducted using available data from the international databases, including PubMed, Google Scholar, Science direct, Cochrane library and unpublished reports. All observational studies reporting the prevalence of latrine utilization in Ethiopia were included. Four authors independently extracted all necessary data using a standardized data extraction format. STATA 13 statistical software was used to analyze the data. The Cochrane Q test statistics and $P$ test were used to assess the heterogeneity between the studies. A random effect model was computed to estimate the pooled level of latrine utilization in Ethiopia. In addition, the association between latrine utilization and the educational level of the users was analyzed.

Results: After reviewing of 1608 studies, 17 studies were finally included in our meta-analysis. The result of 16 studies revealed that the pooled prevalence of latrine utilization level in Ethiopia was 50.02\% (95\%Cl: 40.23, 59.81\%). The highest level (67.4\%) of latrine utilization was reported from Southern Nations Nationality and People regional state, followed by Amhara regional state $(50.1 \%)$. Participants who completed their high school and above education were more likely (OR: $1.79,95 \% \mathrm{Cl}: 1.05,3.05)$ to utilize latrine compared to those who did not attend formal education.

Conclusion: In Ethiopia, only half of the households utilize latrine and the level of utilization has significant association with educational status.

Keywords: Latrine-utilizations, Educational-status, Systematic-review, Meta-analysis, Ethiopia

\footnotetext{
* Correspondence: chertesema@gmail.com

${ }^{1}$ Department of Public Health, College of Health Sciences, Debre Markos

University, Debremarkos, Ethiopia

Full list of author information is available at the end of the article
}

(c) The Author(s). 2018 Open Access This article is distributed under the terms of the Creative Commons Attribution 4.0 International License (http://creativecommons.org/licenses/by/4.0/), which permits unrestricted use, distribution, and reproduction in any medium, provided you give appropriate credit to the original author(s) and the source, provide a link to the Creative Commons license, and indicate if changes were made. The Creative Commons Public Domain Dedication waiver (http://creativecommons.org/publicdomain/zero/1.0/) applies to the data made available in this article, unless otherwise stated. 


\section{Background}

Communicable diseases are serious public health problems, affecting billions of people around the world, mainly the third world countries [1, 2]. Latrine utilization, the main determinant for communicable diseases control, is still at its lower level in developing countries including Ethiopia [3]. Access to safe drinking water and sanitation is a basic necessity that is vital for human health and among the basic human rights declared by the United Nations. Ensuring sanitation demands the availability of facilities and services for the safe disposal of human excreta. It is one of the components of the sustainable development goals that are set to be achieved by 2030 [4-7].

Worldwide, a tremendous progress has been made in increasing access to facilities that ensure hygienic separation of human excreta from human contact. More than half of the global population used basic sanitation services and nearly two out of five people (39\%) utilized safely managed sanitation services. Nevertheless, billions of people still remained without even the basic sanitation services and around 800 million people used unimproved facilities. Most countries are moving off the track to attain the desired coverage for sanitation set in the sustainable development goals [8-10].

In communities where access to improved sanitation facilities is low, people are forced to engage in unsafe practice of open defecation. This practice continues to be a major challenge and about 2.3 billion people who still lack basic sanitation service either practice open defecation (892 million) or use unimproved facilities such as pit latrines without a slab or platform, hanging latrines or bucket latrines (856 million) [11]. In sub-Saharan Africa, the number of people who defecate in the open field rose from 204 to 220 million by 2015 $[8,9,12,13]$. Diarrheal and other communicable diseases are often linked with poor sanitation and open defecation. Moreover, higher rates of open defecation are also associated with significant socioeconomic, environmental and major public health consequences affecting the overall health and dignity of mankind, the most vulnerable groups being women and children [1, 14-19].

Increasing availability and proper utilization of latrines is essential and a cost-effective strategy to overcome disease burden associated with improper excreta management [20-22]. The use of latrines can be affected by a range of behavioral, cultural, social, geographic and economic factors differing across communities [23-29].

According to the 2016 Ethiopian Demographic and Health Surveys report, $56 \%$ of the rural households use unimproved toilet facilities. One in every three households in the country has no toilet facility [30]. The inauguration of the health extension program in 2003 and the national water supply, sanitation and hygiene [31] program contributed much to the improved coverage of latrines across the country. However, achieving real gains in increasing latrine use and quality remained as a challenge [32-35].

In Ethiopia, different fragmented and small studies have been conducted to assess the level of latrine utilization. Nevertheless, the findings of these studies reported highly varying figures. Some of the findings showed as the level of latrine utilization is at a good progress, while some others revealed the awkward aspect. The previous studies also indicated the presence of significant variability in latrine utilization from region to region [36, 37].

Determining the pooled prevalence of latrine utilization at a country level will provide an overall figure with better estimation accuracy. Therefore, this systematic review and meta-analysis was aimed at estimating the pooled prevalence of latrine utilization and its association with education level. The findings from this study will have a paramount importance for decision makers revealing at what level the country is with regard to latrine utilization.

\section{Methods \\ Searching strategies}

This systematic review and meta-analysis was conducted to estimate the pooled prevalence of latrine utilization and its association with educational level of the user in Ethiopia. To conduct this study, all potentially relevant articles, grey literatures, and government reports were meticulously searched. The Preferred Reporting Items of Systematic Reviews and Meta-Analysis (PRISMA) checklist guideline was used to ensure the scientific rigor [38]. We searched articles from international databases including Cochrane library, PubMed, Google Scholar, and Science direct. The reviewers used the following keywords "prevalence", "("toilet facilities"[MeSH Terms] OR ("toilet"[All Fields] AND "facilities"[All Fields]) OR "toilet facilities"[All Fields] OR "latrine"[All Fields]) AND ("utilization"[Subheading] OR "utilization"[All Fields]) AND ("ethiopia"[MeSH Terms] OR "ethiopia"[All Fields]) to get published articles from above mentioned databases.

"The search was carried out from September to October, 2017. All articles published until October, 2017 were included in the review.

\section{Inclusion and exclusion criteria}

The current meta-analysis and systematic review included studies conducted only in Ethiopia and that reported the level of latrine utilization, articles published in the scientific journals and grey literatures. Studies written in English language and full-text articles only were considered. In addition, the review considered all observational study designs (Cross-sectional, case-control, and cohort) reported the level of latrine utilization in Ethiopia. We excluded articles which were not able to be accessed for 
full article text after communicating the principal investigator of the primary studies by email at least three times.

\section{Outcome of interest}

The primary outcome of interest was the pooled prevalence of latrine utilization. The prevalence was computed from the proportion in which the number of individuals who had proper latrine utilization to the total number of households with functional latrine multiplied by 100 . Estimate of the association between educational status and level of latrine utilization was also a second outcome.

\section{Study setting}

This systematic review and meta-analysis was conducted in Ethiopia. The country is located in the Horn of Africa with projected population of 107,421,970 by 2018 year. The country is divided into nine regions and two administrative cities. The regions are Afar, Amhara, Benishangul-Gumuz, Gambella, Harari, Oromia, Southern Nations, Nationalities, and Peoples of Ethiopia, and two city administrates are Addis Ababa and Dire Dawa [39].

\section{Operational definitions}

Improved sanitation facilities (Latrine) are those designed to hygienically separate excreta from human contact. These include wet sanitation technologies (flush and pour flush toilets connecting to sewers, septic tanks or pit latrines) and dry sanitation technologies (ventilated improved pit latrines; pit latrines with slabs; or composting toilets) [11].

Latrine utilization - households with either shared or private functional latrines functional latrines and the family disposed the faeces of under-five children in a latrine, no observable faeces in the compound, no observable fresh faeces on the inner side of the squatting hole and the presence of clear foot-path to the latrine is uncovered with grasses or other barriers of walking [1].

Education categories: The primary studies classified education for the head of the households as 1) not attended formal education, 2) attended primary education $(1-8), 3)$ attended secondary Educations and 4) college and above.

\section{Data abstraction}

Four authors (CTL, AA, AF and HM) independently searched the studies, articles, and reports, and extracted all necessary data using a standardized data extraction format using Microsoft Excel. The extracted parameters were: primary author, publication year, region where the study was conducted, the study design used, sample size, level of latrine utilization, and quality of each study. Then, three authors (AT, GDK and NM) checked the data extraction process. Finally, nine authors (AN, BT,
FW, DJB, GM, YA, GDK, MYB and MS) participated in resolving the disagreement.

\section{Quality assessment of the studies}

We used Newcastle-Ottawa Scale adapted for cross-sectional studies quality assessment to assess the quality of each study [40]. The tool has mainly three sections; the first section grades from five stars and mainly focuses on the methodological quality of each study (sample size, response rate and sampling technique). The second section deals with the comparability of the studies, with a possibility of two stars to be gained. The last section deals with the outcomes and statistical analysis of the original study with a possibility of three stars to be gained (Additional file 1). Two authors independently assessed the quality of each original study. Disagreements between two authors were resolved by taking the mean score of the two authors. Finally, researches with a scale of $\geq 6$ out of 10 were considered as achieving high quality. This cut-off point was declared after reviewing relevant literatures.

\section{Data analysis}

The extracted data were compiled in Microsoft Excel format and analyzed using STATA version 13 statistical software. The binomial distribution formula was used to calculate standard error for each eligible original article. Heterogeneity between studies was assessed using Cochran's Q- statistics and Higgins' and Thompson's $I^{2}$ test [41]. As the preliminary output of the test statistics revealed a significant heterogeneity among studies $\left(I^{2}=99.5 \%\right.$, $p=0.00)$, random Effects meta-analysis model was used for approximation of the Der Simonian and Laird's pooled effect. Subgroup analysis was also performed among regions, study setting and education in relation to the latrine utilization as well as trends of latrine utilization was made. To reduce the random variations between the individual point estimates of the primary study, a subgroup analysis was carried out based on study settings (regions). Possible source of heterogeneity was also identified by Univariate Meta-regression by taking the sample size and year of publication as covariates. Furthermore, Egger and Begg tests at $5 \%$ significant level were employed to assess publication bias [42]. The point prevalence with its corresponding 95\% confidence interval was presented using forest plot. In this forest plot, the size of each individual box revealed the weight of the study, while each crossed line refers to $95 \%$ confidence interval. We conducted log-odd ratio for the second outcome (the relationship between latrine utilization and educational status of the households.

\section{Results}

One thousand six hundred eight (1608) primary studies that addressed latrine utilization and associated factors were searched using both through PubMed, Google 
Scholar, science direct and grey as well as the government reports. Seven hundred twelve (42.3\%) of these identified articles were excluded because of similarity and duplicated articles. Among the remaining 896articles, 543 articles were excluded after reviewing their titles for a reason of relevance for our objective. The rest 353 articles were screened for abstracts and 286 were excluded after reading their abstract sections. Therefore, 71 full-text articles were accessed and assessed for eligibility based on the pre-set criteria, and from these 52 were excluded for not fitting the inclusion criteria. Finally, 19 studies fulfilled the eligibility criteria and included in the final meta-analysis (Fig. 1).

\section{Overview of included studies}

These 19 (of which 2 were unpublished) studies were published from 1999 to 2017. In the current meta-analysis, 966,362 study participants were involved to estimate the pooled prevalence of latrine utilization in Ethiopia in which the lowest (30.99\%) latrine utilization was observed from a study conducted at Akaki, Oromia region [43] while the highest prevalence (99.4\%) was reported from a study conducted in Dembia district of Amhara region [44]. Regarding the study design, all (100\%) of the studies were cross-sectional study designs. The sample sizes of the studies ranged from 355 to 955,985 . This meta-analysis and systematic review used data taken from primary studies of five (5) (Amhara, south nation and nationality people of Ethiopia, Oromia, Tigray and Harari) regions of Ethiopia that shares eight (42\%), $4(21 \%), 4(21 \%), 2(1 \%)$ and 1 $(0.5 \%)$ respectively (see the Additional file 2 ).

\section{Meta-analysis}

As indicated above in the Additional file 2, 19 studies were found to be eligible for the analysis. Of these, two

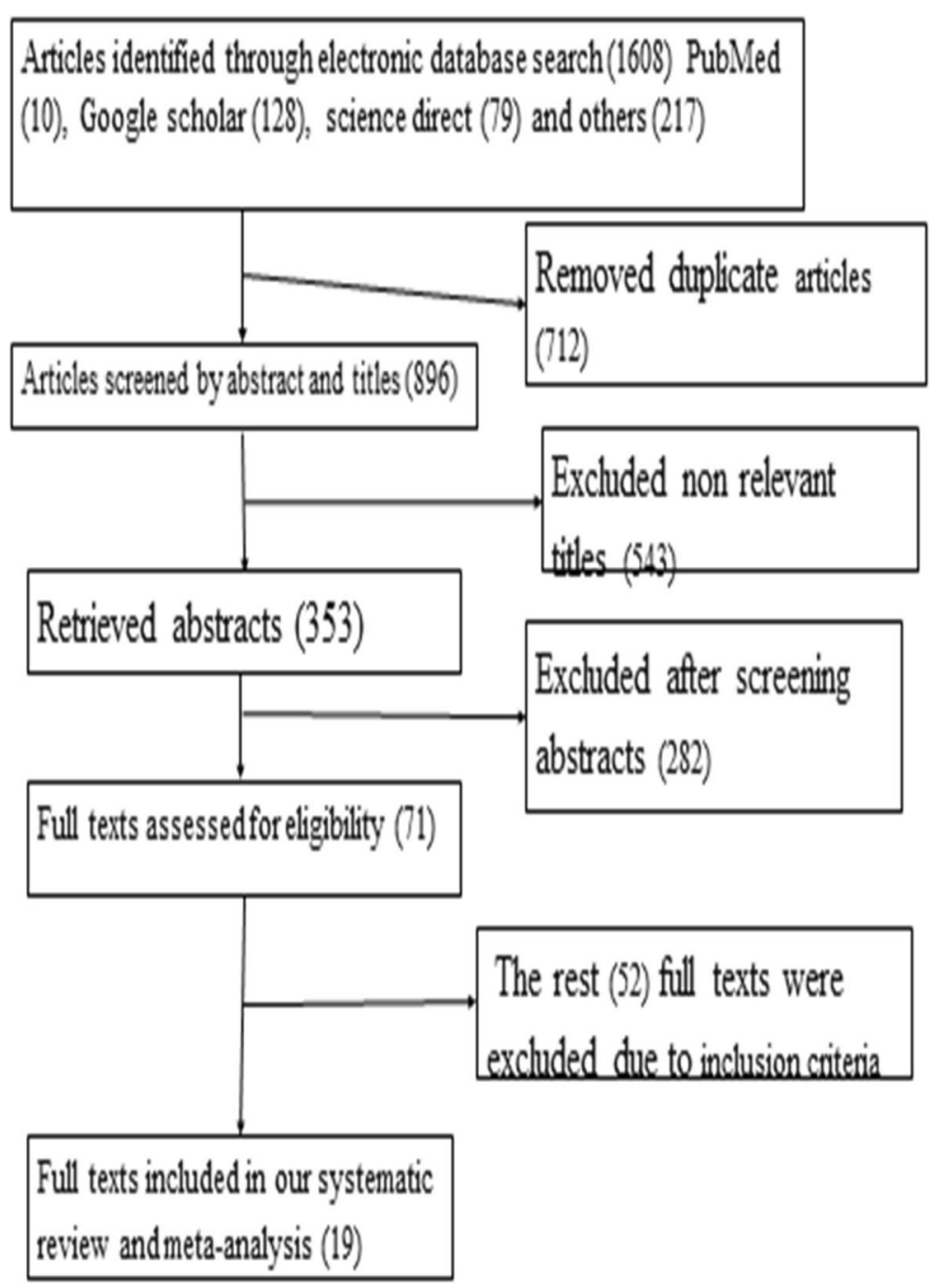

Fig. 1 Flow chart to describe the selection of studies for a systematic review and meta-analysis of the level of latrine utilization and is association with educational status at Ethiopia 
Table 1 Related factors with heterogeneity of the latrine utilization in the current meta-analysis (Based on Univariate Meta Regression)

\begin{tabular}{lll}
\hline Variables & Coefficient & $P$-value \\
\hline Publication year & 1.14 & 0.3 \\
Sample size & -0.00001 & 0.3 \\
\hline
\end{tabular}

studies $[44,45]$ were excluded from forest plot of the pooled level of latrine utilization after we did sensitiy analysis. The sensitivity analysis for Amhara, Tigray, SNNP and others (Oromia and Harar) regions were revealed as $\left(\mathrm{I}^{2}=98.3, p\right.$ value $\left.=0.001\right), \quad\left(\mathrm{I}^{2}=98.9, p\right.$ value $=0.001),\left(\mathrm{I}^{2}=62.7, p\right.$ value $\left.=0.001\right)$ respectively . Seventeen articles were considered to determine the pooled prevalence of latrine utilization in Ethiopia that found to be $50.2 \%$ (95\% CI: 40.23, 59.81\%). High heterogeneity, $\left(\mathrm{I}^{2}=99.5, p\right.$ value $\left.<0.001\right)$, was observed between 17 primary studies included in this review. As a result, to reduce it, we performed a subgroup analysis $\left(\mathrm{I}^{2}=99.5, p\right.$ value $\left.=0.001\right)$ and come up a slight improvement. The regional subgroup analysis revealed that significant regional variation regarding latrine utilization was observed across the country. Southern nation nationalities and people of Ethiopian have better latrine utilization while Oromia utilizes least. As a result, a random effect model was employed to estimate the pooled prevalence of latrine utilization in Ethiopia.

To identify the possible source of heterogeneity, different factors associated with the heterogeneity such as publication year and sample size of the study were investigated by using Univariate meta-regression models, but none of these variables were found to be statistically significant. Even though it is not statistically significant for the increments of both sample size and publication year, as sample size, increase the level of latrine utilization was showed slightly decreased, whereas the proportion showed level of latrine utilization increments as publication years does also (Table 1). Moreover, Publication bias was also assessed using Begg and Egger tests. The result of Begg and Egger tests were not statistically significant for estimating the level of

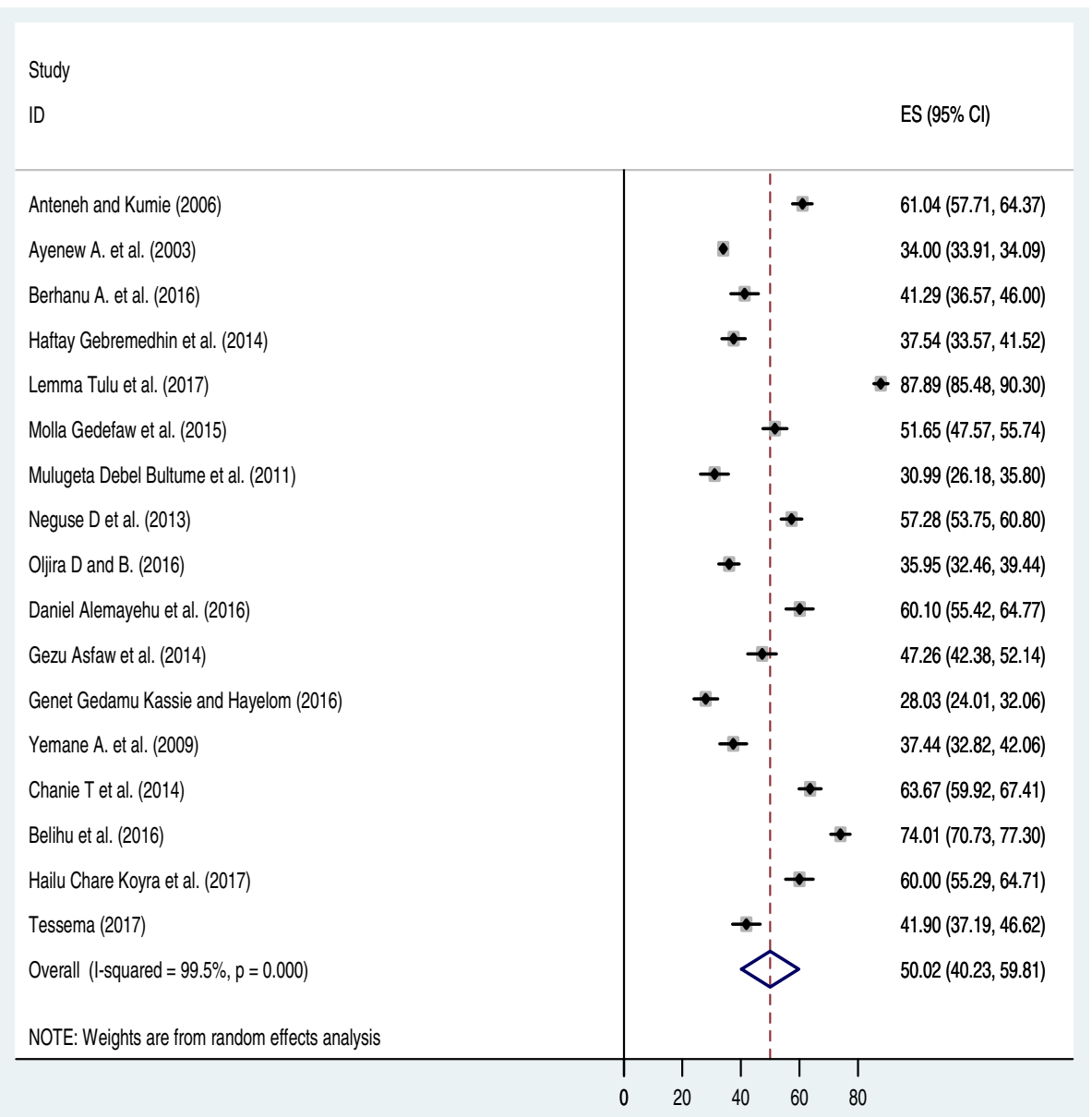

Fig. 2 Forest plot of the pooled prevalence of latrine utilization in Ethiopia 
latrine utilization $(p=0.15)$ and $(p=0.3)$ respectively (Figs. 2, 3, 4).

\section{Subgroup analysis}

In order to appreciate the heterogeneity of individual studies, subgroup analysis was conducted based on the region where the studies were conducted. The output of subgroup analysis revealed that, the highest latrine utilization was observed in south nation and nationalities and peoples of Ethiopia with a prevalence of $67.4 \%$ (95\% CI: $50.3,84.5)$ followed by Amhara with pooled latrine utilization of $50.1 \%$ (95\% CI: 39.7, 62.2). Besides, subgroup analysis based on the sample size $(\geq 500$ and $<500)$ of studies revealed that subgroup of sample size $\geq 500,55.9 \%$ (95\% CI: 40.0, 71.8\%) revealed a higher latrine utilization than the subgroup of sample size $<500,43.4 \%(95 \%$ CI: 34.9, 59.8\%) (Table 2).

Similarly, subgroup analysis was also performed between study settings (urban, rural and both). The pooled latrine utilization for study settings that means rural, both and urban were found to be 49.25(38.48, 60.01), 40.84(33.95, $47.74)$ and $61.85(43.88,79.81)$ respectively (Fig. 5).

\section{The association between latrine utilization and} educational status

A total of 7(41.2\%) studies that fulfilled the inclusion criteria and which were considered for determining the pooled level of latrine utilization assessed the association between education and latrine utilization practice. Only one $(14.3 \%)$ of the studies estimated that education has a negative association with latrine utilization. That is, respondents who are literate are less likely to utilize latrine compared with the illiterate respondents [46].

The remaining $6(85.7 \%)$ of the studies reported that $[44,47-51]$ as people get education they use a latrine (Positive association). The heterogeneity $\left(\mathrm{I}^{2}=90.4 \%\right.$ and $P$-value $<0.001)$ became lower during this subgroup analysis when compared with the pooled latrine use analysis result. However, lower heterogeneity (compared with the pooled results of all 17 studies) was observed during subgroup analysis, a random effect meta-analysis model was employed to determine the association between latrine utilization and educational status of the respondents. The overall effect of educational status (as indicated in this subgroup analysis) showed that individual educational status was

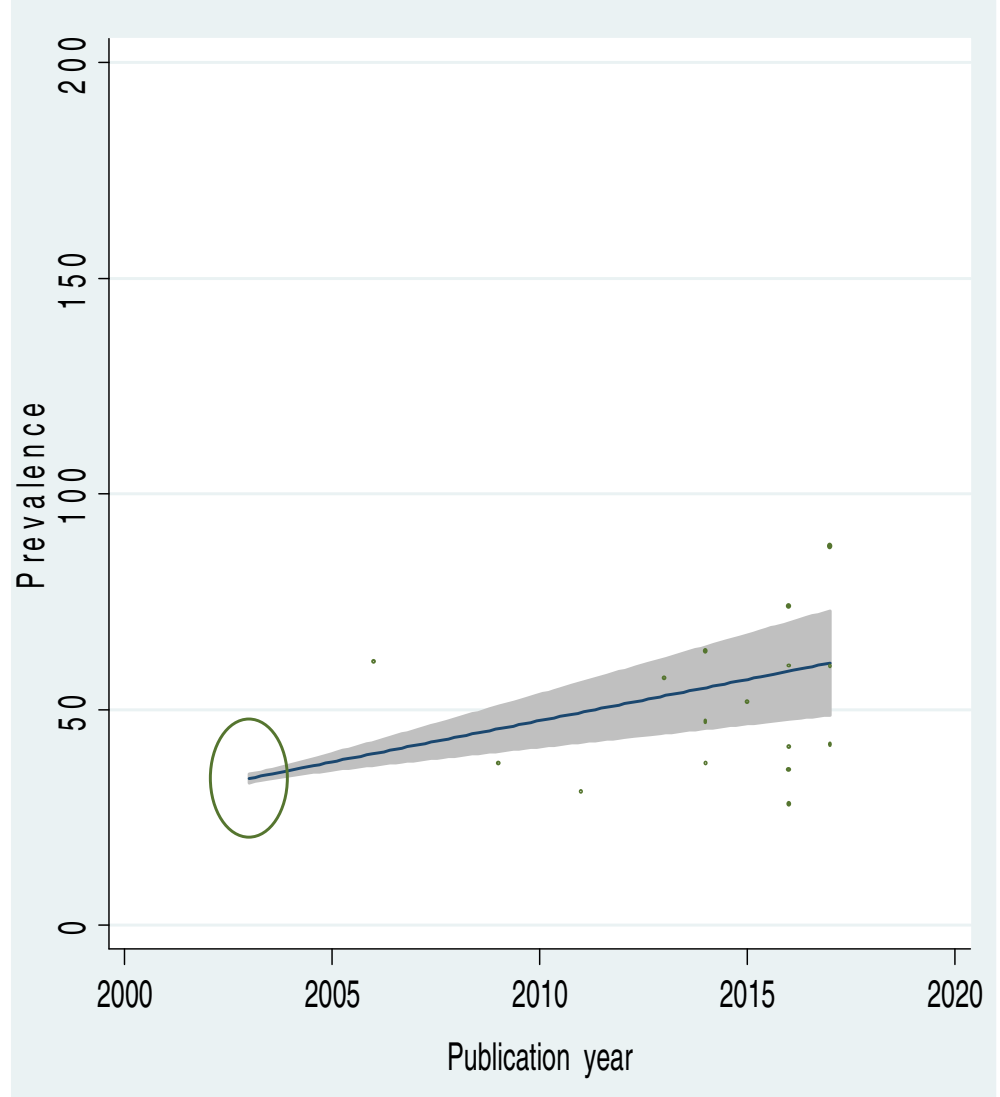

Fig. 3 The Univariate Meta regression to identify possible source of heterogeneity by publication year 


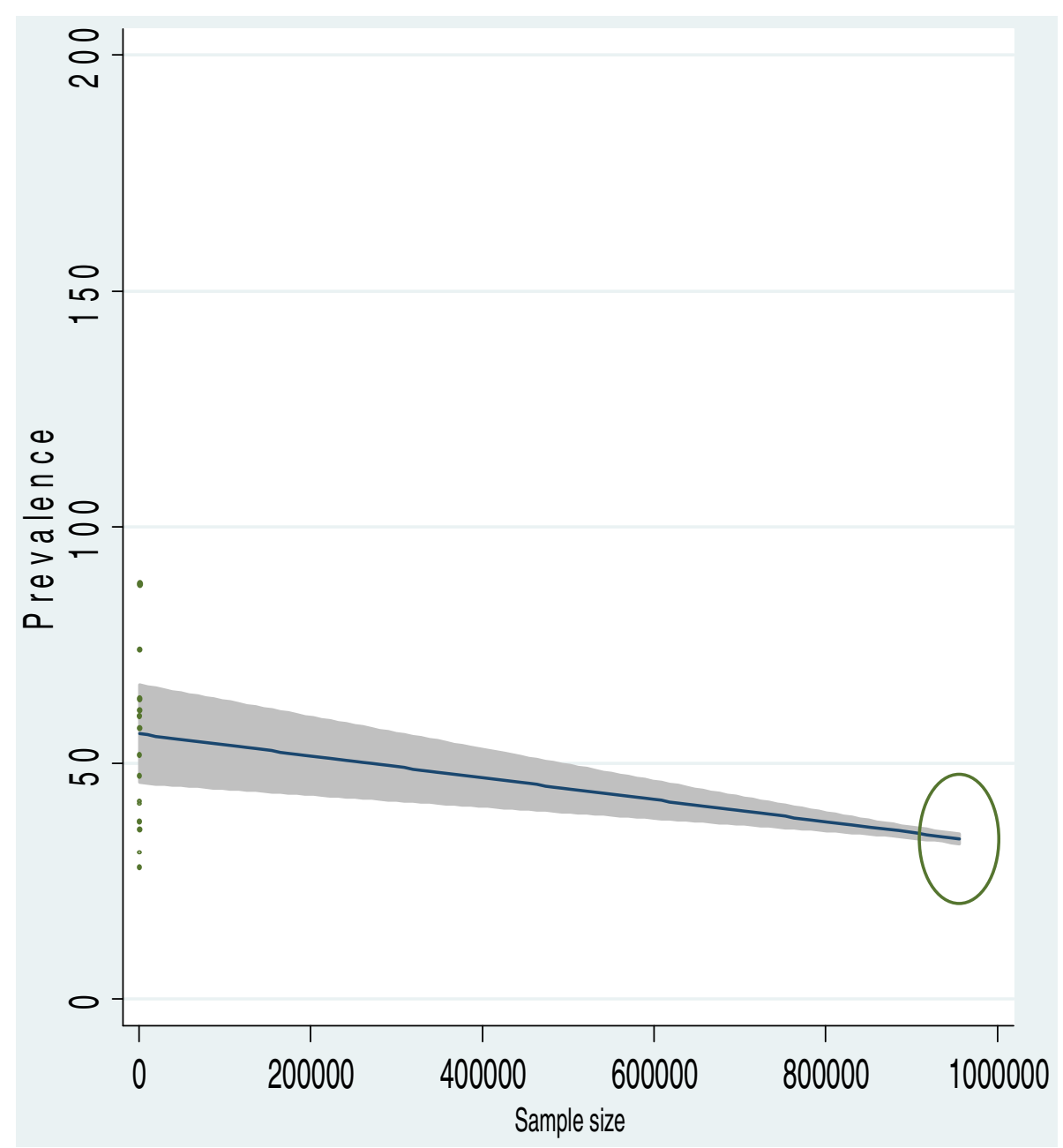

Fig. 4 The Univariate Meta regression to identify possible source of heterogeneity by sample size

significantly associated with latrine utilization (OR: 1.79, 95\%CI: 1.05, 3.05) (Fig. 6).

\section{Discussion}

This systematic review and meta-analysis disclosed that the pooled level of latrine utilization in Ethiopia was $50.0 \%$ (95\% CI: $40.23,59.81 \%)$. This finding is lower than a study conducted in Ghana which revealed that $66.5 \%$ of the community had proper latrine utilization [52]. Similarly, the finding of this meta-analysis is slightly lower that a study conducted in Sub-Saharan African countries revealed that proper latrine utilization was estimated to be 63\%). Likewise, the finding is much lower than a community-based study conducted in Nepal

Table 2 Subgroup prevalence of latrine utilization among regions of Ethiopian country, 2017 ( $n=17)$

\begin{tabular}{lllll}
\hline Variables & Characteristics & Number of studies included & Sample size & Estimate (95\% Cl) \\
\hline By Region & Amhara & 6 & 3350 & $50.1(39.7,62.2)$ \\
& Tigray & 4 & 957,733 & $41.5(30.9,52.2)$ \\
& SNNPE & 4 & 2204 & $67.4(50.3,84.5)$ \\
By sample size & Others & 3 & 1501 & $36.3(30.7,41.9)$ \\
& $\geq 500$ & 9 & 3332 & $55.9(40.0,71.8)$ \\
Overall & $<500$ & 8 & 961,456 & $43.4(34.9,59.8)$ \\
\hline
\end{tabular}




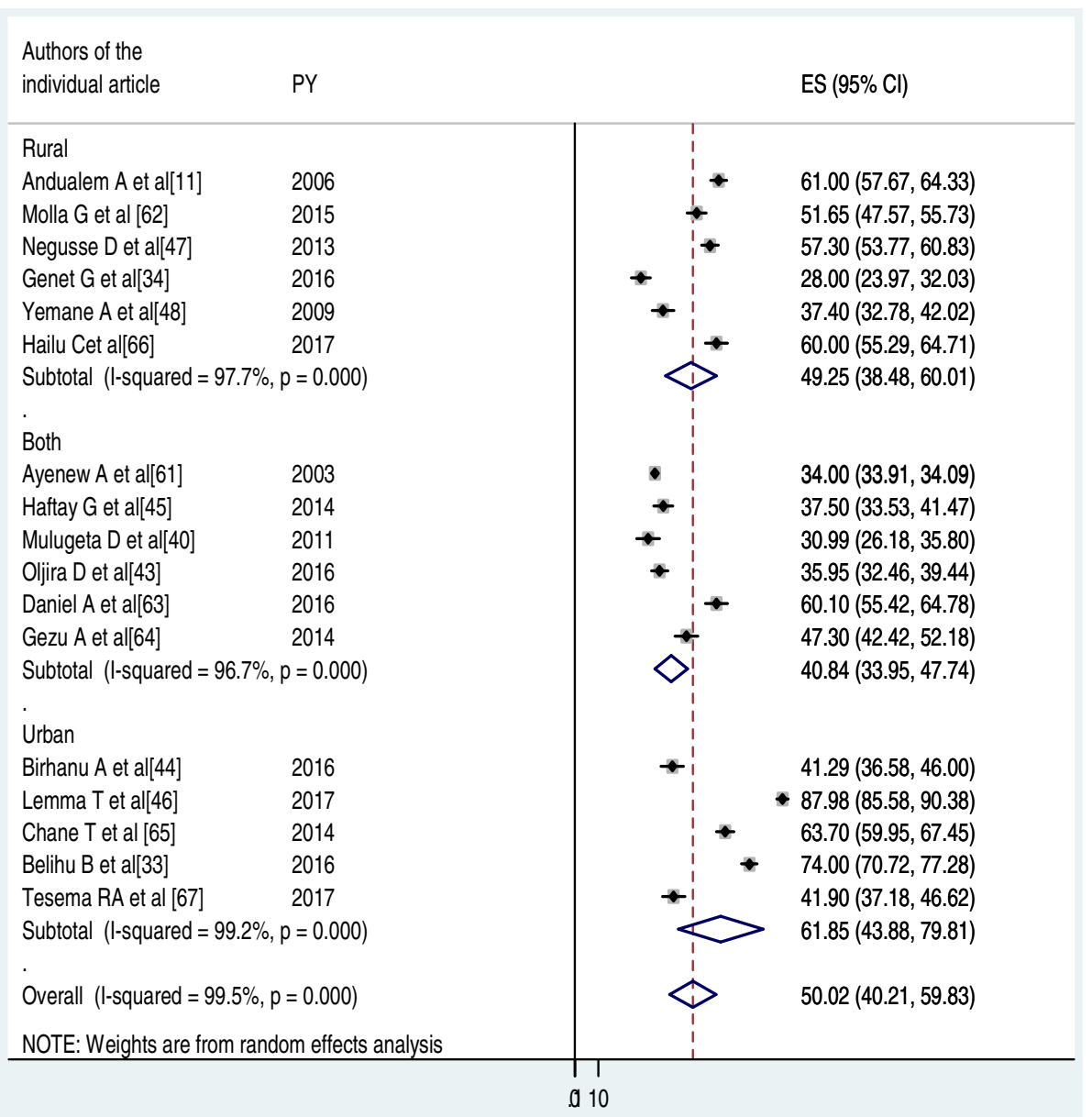

Fig. 5 The subgroup analysis of latrine utilization status by study settings (rural, both urban and rural, and urban) in Ethiopia

(94.3\%) [53]. The possible explanation for the above-observed discrepancy between the current meta-analysis and comparable findings might be due to the difference in the Sociodemographic characteristics of the study participants. A report from other sub-Saharan African countries contained a data mostly collected from the urban population while in this study; both urban and rural settings were considered. The other possible explanation for the above variation could be due to the difference in study design.

However, the current pooled analysis of latrine utilization is higher than a the world health organization report (39\%) [10]. Similarly, the current pooled latrine utilization result is slightly higher than from southern Asia countries reported by the world health organization [54] and Indian where only $47 \%$ of the respondents use latrine always [55]. The observed discrepancy could be resulted from time, study setting, sample size and socioeconomic difference among the different settings. The additional possible justification for the discrepancy might be because of half the population of in developing world lacks basic sanitation [56]. In addition, in Ethiopia, the government has been implementing different interventions to improve the level of latrine utilization (basic sanitation) for example, the implementation of health extension package since 2003. The health policy (focused on prevention of diseases and promotion of health using the provision of basic sanitation in all level of the country) of Ethiopia could be also another determinant factor for the slight improvement of the latrine utilization level in the country [57].

The pooled prevalence of latrine utilization level in Southern Nation and nationalities and People region of Ethiopia (SNNRPE) was $67.4 \%$ which is higher than the pooled prevalence of latrine utilization level than other regions of the country Ethiopia; in Amhara 50.1\%, in Tigray $41.5 \%$ and others $36.3 \%$. The possible explanations for this variation might be due to the difference in socioeconomic and sociocultural difference between the regions. The other possible explanation for this variation might be due 


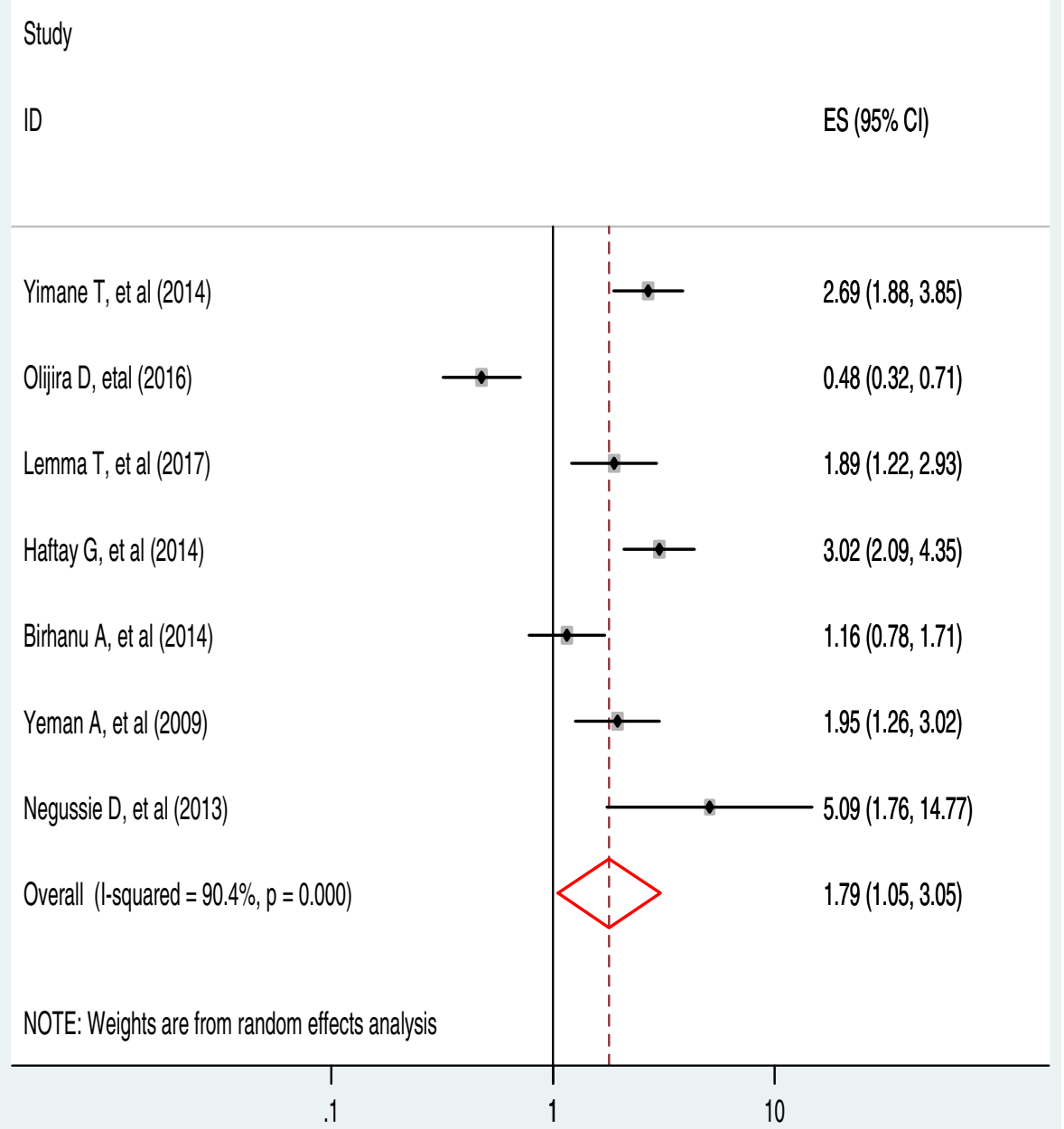

Fig. 6 The pooled odds ratio of the association between latrine utilization and educational status in Ethiopia

to differences in the study period in which data collection period for all studies taken from the SNNRPE is recent than the others. On top of the above possible justification, almost half of the studies conducted at south nation nationality and people of Ethiopia were conducted in urban set-up including the capital city of the region. In addition, from this subgroup analysis, it was observed that the estimated latrine utilization level in the SNNRPE was $64.7 \%$, which is higher than the estimated report on latrine utilization by the Min Ethiopian demographic health survey 2014 which was $54 \%$ [58] of the community use latrine. The subgroup analysis also revealed that latrine utilization was better in Amhara region next to the SNNRPE followed by Tigray. This finding was directly related with the educational development of the regions. Currently, the quality of education reported better at $\mathrm{Ti}$ gray, Amhara region, Oromia and SNNPE, while bringing quality education on the rest regions are still challenging due to their living style. People living other than these detailed above lives a nomadic and pastoral life.

In this systematic review and meta-analysis, we performed a subgroup analysis by study settings (urban, both setting and rural). However, the finding was not statistically significant despite a slight discrepancy. Latrine utilization was found to be better in the urban setting $(61.85 \%)$ as compared to rural (49.25\%) and both (40.84\%). Better latrine utilizations in urban setting might be due to high literate populations reside urban than the counterpart settings.

Educational level of the respondents has a significant association with latrine utilization. The finding of this study is supported by other similar study conducted on the impact of sanitation intervention on latrine coverage and uses a worldwide report that means education level has an effect on the community latrine utilization [59]. This might be due to that 
education has a significant influence on human behavior towards behaving health activities. Similarly as peoples' educational status increases, their knowledge on the diseases causation, transmission and the role of human waste to the occurrence of communicable diseases increases. Therefore, to keep their health well they manage and dispose of every type of wastes (including human excreta) safely wherein properly constructed latrine. On the contrary to this study, educational status of the respondents (head of the household) has no any significant association with latrine utilization in one study conducted in Nepal [60]. This might be due to the fact that even though slightly more than half of the participates were illiterate (51.7\%), the government of Nepal is committed to improving sanitation throughout the country, one priority campaign is improving latrine coverage towards attaining open defecation free areas all over the country by 2017 [17]. Despite the fact that a lot activities and strategies(like training manpower, ONE WASH, Health Extension Package and Community Lead Total Sanitation and Hygiene Behavioral Change) have been conducted in the country Ethiopia, latrine utilization was remain on half of the country vision which was $100 \%$ basic sanitation (including proper latrine utilization) [61].

Education and creating awareness is one among the 16 packages included in the health extension packages. Health extension workers employed to implement this packages provide a routine health education to improve the community awareness to increase latrine utilizations [62]. This implies that as the educational level of individual increased latrine utilization will increase.

\section{Limitations of the study}

In this systematic review and meta-analysis, we recognized some limitations. The first concern was the use of only English language articles as inclusion criteria. The other constraint is the cross sectional nature of the included articles, which can affect the second objective due to the presence of confounders. In addition, the pooled prevalence might not represent the whole country as the included articles were only from six administrative regions.

\section{Conclusion}

Only half of the community has had latrine utilization practice and which is lower compared with the country target $100 \%$ set to be met by 2015 . This meta-analysis also showed that educational status of the community has a significant association with latrine utilization; that is, attending formal education is a positive predictor for community latrine utilization.

\section{Additional files}

Additional file 1: Quality assessment of 19 included studies. (DOCX $17 \mathrm{~kb}$ )

Additional file 2: Descriptive summary of 19 studies included in the meta-analysis of the level of latrine utilizations and its association with educational status in Ethiopia. (DOCX $22 \mathrm{~kb}$ )

\section{Abbreviations}

EDHS: Ethiopian Demographic and Health Survey; MDGs: Millennium Development Goals; PRISMA: Preferred Reporting of Systematic Reviews and Meta-Analysis; SNNRE: Southern Nation and Nationalities People region of Ethiopia; WASH: Water Sanitation and hygiene

\section{Acknowledgements}

The authors of this work would like to forward great and deepest gratitude for Debremarkos University for creating convenient environment and internet service. Last but not least, we would like to forward our acknowledgement for Dr. Belete Tafesse, who is a fluent in English and experienced academics in editing a manuscript for his time spent and willingness to edit this manuscript and made the necessary revision.

\section{Funding}

No funding was obtained for this study.

Availability of data and materials

Data will be available upon request of the corresponding author.

\section{Authors' contributions}

CTL: Conception of research protocol, study design, literature review, data extraction, data analysis, interpretation and drafting the manuscript. AA, AN, GM, AT, HM, BT, NM, FW, YAB, AF, MS, GDA, DJB, MYB and GDK: data extraction and quality assessment, data analysis and reviewing manuscript. MYB: revised the entire section of the final manuscript critically. And also he gave final approval of the version to be published. All authors have read and approved the manuscript.

Ethics approval and consent to participate

Not applicable.

Consent for publication

Not applicable.

Competing interests

The authors declare that they have no competing interests.

\section{Publisher's Note}

Springer Nature remains neutral with regard to jurisdictional claims in published maps and institutional affiliations.

\section{Author details}

'Department of Public Health, College of Health Sciences, Debre Markos University, Debremarkos, Ethiopia. ${ }^{2}$ Department of Nursing, College of Health Sciences, Debre Markos University, Debremarkos, Ethiopia. ${ }^{3}$ Department of Human Nutrition and Food Science, College of Health Sciences, Debre Markos University, Debremarkos, Ethiopia. ${ }^{4}$ Department of Medical Laboratory technology, College of Health Sciences, Debre Markos University, Debremarkos, Ethiopia. ${ }^{5}$ Department of Biomedical Science, School of Medicine, Debre Markos University, Debremarkos, Ethiopia.

Received: 1 March 2018 Accepted: 4 July 2018

Published online: 20 July 2018

\section{References}

1. Anteneh A, Kumie A. Assessment of the impact of latrine utilization on diarrhoeal diseases in the rural community of Hulet Ejju Enessie Woreda, East Gojjam Zone, Amhara region. Ethiop J Health Dev. 2010;24(2)

2. Ammar FJH: Identifying and supporting the most disadvantaged people in CLTS: a case study of Bangladesh.. 2010. 
3. Ethiopia briefing: Economic impact of water and sanitation - unicef, Sanitation and Water for all. 2011.

4. Wateraid sf, [cited on oct 09 2017]; Available form: www.wateraid.org/ publications.

5. Curtis V, Cairncross S. Effect of washing hands with soap on diarrhoea risk in the community: a systematic review. Lancet Infect Dis. 2003;3(5):275-81.

6. Demeke A, Abebe A, Wale A, Kaba E, Sime E, Addis H, Admassu S, Steenhuis T. Sanitation promotion and household latrine, November 2011. (accessible at website: https://wateraidethiopia.org)

7. Assembly UG: The human right to water and sanitation UN resolution 2010, 64:292 2010.

8. Organization WH. Progress on sanitation and drinking water: 2015 update and MDG assessment: World Health Organization; 2015.

9. Organization WH, UNICEF: progress on drinking water, sanitation and hygiene: 2017 update and SDG baselines. 2017.

10. Supply WUJW, Programme SM. Progress on drinking water and sanitation: 2014 update: World Health Organization; 2014

11. Progress on Drinking Water, Sanitation and Hygiene. 2017 Update and SDG Baselines. Geneva: World Health Organization (WHO) and the United Nations Children's Fund (UNICEF). Licence: CC BY-NC-SA 3.0 IGO; 2017.

12. Galan DI, Kim S-S, Graham JP. Exploring changes in open defecation prevalence in sub-Saharan Africa based on national level indices. BMC Public Health. 2013;13(1):527.

13. UNICEF: Water,sanitation and hygiene, annual results report 2016; [cited 9th oct 2017], available form: https:/www.unicef.org/publicpartnerships/files/ 2016arr_wash.pdf. 2016.

14. Bartram J, Cairncross S. Hygiene, sanitation, and water: forgotten foundations of health. PLoS Med. 2010;7(11):e1000367.

15. Eshete N, Beyene A, Terefe G. Implementation of community-led Total sanitation and hygiene approach on the prevention of diarrheal disease in Kersa District, Jimma Zone Ethiopia. Sci J Public Health. 2015;3(5):669-76.

16. Karn SK, Harada H. Field survey on water supply, sanitation and associated health impacts in urban poor communities-a case from Mumbai City, India. Water Sci Technol. 2002;46(11-12):269-75.

17. Mara D, Lane J, Scott B, Trouba D. Sanitation and health. PLoS Med. 2010; 7(11):e1000363.

18. Prüss-Ustün A, Bartram J, Clasen T, Colford JM, Cumming O, Curtis V, Bonjour S, Dangour AD, De France J, Fewtrell L. Burden of disease from inadequate water, sanitation and hygiene in low-and middle-income settings: a retrospective analysis of data from 145 countries. Tropical Med Int Health. 2014;19(8):894-905.

19. Ziegelbauer K, Speich B, Mäusezahl D, Bos R, Keiser J, Utzinger J. Effect of sanitation on soil-transmitted helminth infection: systematic review and meta-analysis. PLoS Med. 2012;9(1):e1001162.

20. Clasen TF, Bostoen K, Schmidt WP, Boisson S, Fung ICH, Jenkins MW, Scott $B$, Sugden $S$, Cairncross $S$. Interventions to improve disposal of human excreta for preventing diarrhoea. Cochrane Libr. 2010;

21. Hulton G, Organization WH: Global costs and benefits of drinking-water supply and sanitation interventions to reach the MDG target and universal coverage. 2012.

22. Hutton G, Haller L, Water S, Organization WH: Evaluation of the costs and benefits of water and sanitation improvements at the global level. 2004.

23. Gebremedhin. Latrine utilization and associated factors in south east zone of tigray region, north ethiopia. Eur J Biomed Pharmac Sci. 2016;3(6):120-6.

24. Asfaw G, Molla E, Vata PK. Assessing privy (Latrine's) utilization and associated factors among households in Dilla town, Ethiopia. Int J Health Sci Res. 2015;5(6):537-44.

25. Budhathoki SS, Shrestha G, Bhattachan M, Singh SB, Jha N, Pokharel PK. Latrine coverage and its utilisation in a rural village of eastern Nepal: a community-based cross-sectional study. BMC Res Notes. 2017;10(1):209.

26. Debesay N, Ingale L, Gebresilassie A, Assefa H, Yemane D. Latrine Utilization and Associated Factors in the Rural Communities of Gulomekada District, Tigray Region, North Ethiopia, 2013: A Community Based Cross-Sectional Study. J Community Med Health Educ. 5:338.

27. Gedefaw M, Amsalu Y, Tarekegn M, Awoke W. Opportunities, and challenges of latrine utilization among rural communities of Awabel District, Northwest Ethiopia, 2014. Open J Epidemiol. 2015;5(02):98.

28. Koyra HC, Sorato MM, Unasho YS, Kanche ZZ. Latrine utilization and associated factors in rural Community of Chencha District, southern Ethiopia: a community based cross-sectional study. Amer J Public Health Res. 2017;5(4):98-104.
29. Yimam YT, Gelaye KA, Chercos DH. Latrine utilization and associated factors among people living in rural areas of Denbia district, Northwest Ethiopia, 2013, a cross-sectional study. Pan Afric Med J. 2014;18

30. Ethiopia Demographic and Health Survey 2016 report.

31. UNICEF W, sanitation and hygiene, annual results report 2016; [cited 9th oct 2017], available form: https://www.unicef.org/publicpartnerships/files/ 2016arr_wash.pdf.

32. Gebru T, Taha M, Kassahun W. Risk factors of diarrhoeal disease in underfive children among health extension model and non-model families in Sheko district rural community, Southwest Ethiopia: comparative crosssectional study. BMC Public Health. 2014;14(1):395.

33. King JD, Endeshaw T, Escher E, Alemtaye G, Melaku S, Gelaye W, Worku A, Adugna M, Melak B, Teferi T. Intestinal parasite prevalence in an area of Ethiopia after implementing the SAFE strategy, enhanced outreach services, and health extension program. PLoS Negl Trop Dis. 2013;7(6):e2223.

34. Tibebu T: Evaluation The Impact Of World Vision Ethiopia, Water, Sanitation And Hygiene Project On The Community: The Case Of Amhara Region, West Gojam Zone, Jabi Tehnane Woreda. St. Mary's University; 2016.

35. Workie NW, Ramana GN: The health extension program in Ethiopia. 2013.

36. Belihu B, S Mohammed, W Godana: Latrine utilization and associated factors among households in Melo Koza District, Southern Ethiopia: A Comparative Cross-Sectional Study. 2012.

37. Kassie GG, Hayelom DH. Assessment of water handling and sanitation practices among rural communities of Farta Woreda, Northwest Ethiopia. Amer J Health Res. 2017;5(5)

38. Liberati A, Altman DG, Tetzlaff J, Mulrow C, Gotzsche PC, loannidis JP, Clarke M, Devereaux PJ, Kleijnen J, Moher D. The PRISMA statement for reporting systematic reviews and meta-analyses of studies that evaluate health care interventions: explanation and elaboration. J Clin Epidemiol. 2009;62(10):e1-34.

39. Worldatlas: Where Is Ethiopia? 2015.

40. Newcastle: Newcastle-Ottawa Scale customized for cross-sectional studies In. available from https://static-content.springer.com/esm/../12889_2012_ 5111_MOESM3_ESM.doc. 2012.

41. Rücker G, Schwarzer G, Carpenter JR, Schumacher M. Undue reliance on I 2 in assessing heterogeneity may mislead. BMC Med Res Methodol. 2008;8:79.

42. Borenstein M, Hedges LV, Higgins J, Rothstein HR. A basic introduction to fixed-effect and random-effects models for meta-analysis. Res Synt Methods. 2010;1(2):97-111.

43. Mulugeta Debel Bultume, David Sanders, D. HM: Utilization of the Health Extension program Services in Akaki District, Ethiopia. 2011.

44. Yimam T, Kassahun A. DH. C: latrine utilization and associated factors among people living in rural areas of Denbia district, Northwest Ethiopia, 2013, a cross-sectional study. Pan African Medical Journal. 2014;

45. Sahlu C, Worku A, M. H: Latrine Utilization and Associated Factors in Rural Community of Amhara Region, Ethiopia: A Community Based CrossSectional Study. 2016.

46. Oljira D, B. T: latrine use and determinant factors in Southwest Ethiopia.. Journal of Epidemiology and Public Health Rev 2016, 1(6).

47. Berhanu A, Muluken A. B. G: latrine access and utilization among people with limited mobility: a cross sectional study.. Archives of. Public Health. 2016;74(9)

48. Haftay Gebremedhin, Teame Abay, Tesfay Gebregzabher, Dejen Yemane, Gebremedhin Gebreegziabiher, S. B: Latrine utilization and associated factors in south East Zone of Tigray region, North Ethiopia. European Journal of Biomedical and Pharmaceutical Sciences 2016, 3(6).

49. Lemma Tulu AK, Hawas SB, Demissie HF, Segni MT. latrine utilization and associated factors among kebeles implementing and non implementing Urban Community led Total sanitation and hygiene in Hawassa town, Ethiopia. Afr J Environ Sci Technol. 2017;11(3)

50. Neguse D, Lalit i, Azeb G, HDY A. latrine utilization and associated factors in the rural communities of Gulomekada District, Tigray region, North Ethiopia, 2013: a community based cross-sectional study. Community Medicine \& Health Educ. 2015;5(2)

51. Yemane A, Hardeep Rai S, Kassahun AKG. Latrine use among rural households in northern Ethiopia: a case study in Hawzien district. Tigray International Journal of Environmental Studies. 2013;70(40)

52. Okechukwu Ol, Okechukwu AA, Noye-Nortey H, Owusu-Agyei. toilet practices among the inhabitants of Kintampo District of northern Ghana. J Med and Med Sciences. 2012;3(8):524.

53. Anne N, Charles BN, Nyenje PM, Robinah NK, BTKF J. Are pit latrines in urban areas of Sub-Saharan Africa performing? A review of usage, filling, 
insects and odor nuisances. BMC Public Health. 2016;16(120) https://doi.org/ 10.1186/s12889-016-2772-z.

54. The Millennium Development Goals Report asdf United States. 2015.

55. Sharmani B, Parimita R, Fiona M, Rachel P, Sophie B, Antara SCT: Impact of Indian Total Sanitation Campaign on LatrineCoverage and Use: A CrossSectional Study in OrissaThree Years following Programme Implementation.

56. The Millennium Development Goals Report 2017.

57. Federal Democratic Republic of Ethiopia Ministry of Health: Health Sector Development Programme IV 2010/11-2014/15. FINAL DRAFT October 2010, version 19 March.

58. Report Ethiopia Demographic and Health Survey 2014

59. OV. Garna, GD. Sclar a, MC. Freemana, G Penakalapati a, KT. Alexander a, P Brooks a, EA. Rehfuess b, S Boissonc, KO. Medlicott c, TF. Clasena: The impact of sanitation interventions on latrine coverage and latrine use: A systematic review and meta-analysis 2013.

60. SS Budhathoki, M Bhattachan, SB Singh, N Jha, APK O: Latrine coverage and its utilisation in a rural village of Eastern Nepal: a community-based crosssectional study.

61. Federal Democratic Republic of Ethiopia Ministry of Health: Ministry of Health National Hygiene and Sanitation Strategy Ethiopia. 2004.

62. packages. HE. Health extension and education center Federal Ministry of Health Addis Ababa. In: Ethiopia; 2007.

Ready to submit your research? Choose BMC and benefit from:

- fast, convenient online submission

- thorough peer review by experienced researchers in your field

- rapid publication on acceptance

- support for research data, including large and complex data types

- gold Open Access which fosters wider collaboration and increased citations

- maximum visibility for your research: over $100 \mathrm{M}$ website views per year

At BMC, research is always in progress.

Learn more biomedcentral.com/submissions 